\title{
Dynamical Casimir Effect and State Transfer in the Ultrastrong Coupling Regime ${ }^{+}$
}

\author{
Giuliano Benenti ${ }^{1,2,3, *(\mathbb{D})}$, Michele Stramacchia ${ }^{1,2}$ and Giuliano Strini ${ }^{4}$ \\ 1 Center for Nonlinear and Complex Systems, Dipartimento di Scienza e Alta Tecnologia, \\ Università degli Studi dell'Insubria, via Valleggio 11, 22100 Como, Italy; mstramacchia@uninsubria.it \\ 2 Istituto Nazionale di Fisica Nucleare, Sezione di Milano, via Celoria 16, 20133 Milano, Italy \\ 3 NEST, Istituto Nanoscienze-CNR, I-56126 Pisa, Italy \\ 4 Department of Physics, University of Milan, via Celoria 16, 20133 Milano, Italy; giuliano.strini@mi.infn.it \\ * Correspondence: giuliano.benenti@uninsubria.it \\ + Presented at the 11th Italian Quantum Information Science conference (IQIS2018), Catania, Italy, \\ 17-20 September 2018.
}

Received: 31 October 2018; Accepted: 23 January 2019; Published: 20 June 2019

check for updates

\begin{abstract}
The dynamical Casimir effect (DCE) manifests itself in the ultrastrong matter-field coupling (USC) regime, as a consequence of the nonadiabatic change of some parameters of a system. We show that the DCE is a fundamental limitation for standard quantum protocols based on quantum Rabi oscillations, implying that new schemes are required to implement high-fidelity ultrafast quantum gates. Our results are illustrated by means of a paradigmatic quantum communication protocol, i.e., quantum state transfer.
\end{abstract}

Keywords: quantum networks; light-matter interaction; quantum Rabi model

\section{Introduction}

High-speed manipulation of quantum systems is vital for the prospects of quantum information processing. In quantum computation, quantum gates should operate on a time scale much smaller than the decoherence time to allow fault-tolerant architectures. In quantum communication, achieving high transmission rate is fundamental to boost applications of quantum cryptography. Circuit quantum electrodynamics (CQED) might play a prominent role to speed up quantum protocols, since it allows one to experimentally address the USC regime of light-matter interaction, where the coupling strength $g$ becomes comparable to, or even exceeds the resonator frequency $\omega[1,2]$.

On the other hand, the performance of quantum protocols operating in the USC regime might be significantly deteriorated, even in the absence of decoherence mechanisms, by the DCE [3]. DCE is the generation of photons from the vacuum due to time-dependent boundary conditions or, more generally, to the nonadiabatic variation of some parameters of a system (for reviews on the DCE see [4,5], while a list of recent papers investigating various aspect of this phenomenon includes [6-18]). This latter case is expected to be relevant in the quest for ultrafast quantum protocols, requiring ultrafast driving of a quantum system.

Here, we demonstrate the strong impact of photon emission by the DCE on a paradigmatic quantum protocol, that is, state transfer from qubit $1\left(Q_{1}\right)$ to qubit $2\left(Q_{2}\right)$, mediated by a single mode of the quantized electromagnetic field (cavity mode $C$ ). The cavity acts as a quantum-bus, which allows to reliably move quantum information and share entanglement between different units of a quantum computing architecture [19-22]. 


\section{Model}

The qubits-cavity dynamics is described by the quantum Rabi Hamiltonian [23-25], with switchable couplings:

$$
\begin{gathered}
H(t)=H_{0}+H_{I}(t), \\
H_{0}=-\frac{1}{2} \sum_{k=1}^{2} \omega_{k} \sigma_{z}^{(k)}+\omega\left(a^{\dagger} a+\frac{1}{2}\right), \\
H_{I}(t)=\sum_{k=1}^{2} f_{k}(t)\left[g_{k} \sigma_{+}^{(k)}\left(a^{\dagger}+a\right)+g_{k}^{\star} \sigma_{-}^{(k)}\left(a^{\dagger}+a\right)\right],
\end{gathered}
$$

where we set $\hbar=1, \sigma_{i}^{(k)}(i=x, y, z)$ are the Pauli matrices for qubit $Q_{k}(k=1,2), \sigma_{ \pm}^{(k)}=\frac{1}{2}\left(\sigma_{x}^{(k)} \mp i \sigma_{y}^{(k)}\right)$ are the rising and lowering operators for the two-level system: $\sigma_{+}^{(k)}|g\rangle_{k}=|e\rangle_{k}, \sigma_{+}^{(k)}|e\rangle_{k}=0, \sigma_{-}^{(k)}|g\rangle_{k}=$ $0, \sigma_{-}^{(k)}|e\rangle_{k}=|g\rangle_{k}$; the operators $a^{\dagger}$ and $a$ create and annihilate a photon: $a^{\dagger}|n\rangle=\sqrt{n+1}|n+1\rangle$, $a|n\rangle=\sqrt{n}|n-1\rangle,|n\rangle$ being the Fock state with $n$ photons. The switching on/off of the couplings is governed by the functions $f_{k}(t)$, in the manner detailed below. For simplicity's sake, we consider the resonant case $\left(\omega_{1}=\omega_{2} \equiv \omega\right)$ and the coupling strengths $g_{1}=g_{2} \equiv g \in \mathbb{R}$. The rotating-wave approximation (RWA) is obtained when we neglect the terms $\sigma_{+}^{(k)} a^{\dagger}$, which simultaneously excites $\mathrm{Q}_{k}$ and creates a photon, and $\sigma_{-}^{(k)} a$, which de-excites $\mathrm{Q}_{k}$ and annihilates a photon. In this limit, Hamiltonian (1) reduces to the Jaynes-Cummings Hamiltonian [23,24], with a switchable coupling. We set $\omega=1$, so that in the RWA the swapping time needed to transfer an excitation from one qubit to the field or vice versa $\left(|e\rangle_{k}|0\rangle \leftrightarrow|g\rangle_{k}|1\rangle\right)$ is $\tau=\pi / 2 g$ and the vacuum Rabi frequency $\Omega=g$. The RWA approximation is a good approximation when $g / \omega \ll 1$ but fails in the USC regime. We work in the interaction picture, where the effective Hamiltonian at resonance is given by $\tilde{H}(t)=e^{i H_{0} t} H_{I}(t) e^{-i H_{0} t}$ (we will omit the tilde from now on).

\section{Rabi-Type State-Transfer Protocol}

In order to transfer a generic pure state $|\psi\rangle=\alpha|g\rangle+\beta|e\rangle$ from qubit $\mathrm{Q}_{1}$ to qubit $\mathrm{Q}_{2}$, we consider the following quantum protocol, based on quantum Rabi oscillations. We first discuss the protocol within RWA, where the state transfer is exact. Initially, $Q_{1}$ is prepared in the state $|\psi\rangle$, while $Q_{2}$ and the cavity mode $C$ are in their ground state. Then $Q_{1}$ interacts with $C$, for a time $\tau$, so that the cavity is at the end in the state $|\tilde{\psi}\rangle=\alpha|0\rangle-i \beta|1\rangle$ and $\mathrm{Q}_{1}$ in $|g\rangle$. The coupling of $\mathrm{Q}_{1}$ with $\mathrm{C}$ is then switched off and $\mathrm{Q}_{2}$ interacts with $\mathrm{C}$, for a time $\tau$. As a result, the state of $\mathrm{Q}_{2}$ is driven to $|\hat{\psi}\rangle=\alpha|g\rangle-\beta|e\rangle$, while $\mathrm{C}$ is left in $|0\rangle$. The transfer of state $|\psi\rangle$ to qubit $Q_{2}$ is recovered after a rotation through an angle $\pi$ about the $z$ axis of the Bloch sphere for that qubit. When the terms beyond the RWA are taken into account, state transfer is no longer perfect and the final state of $Q_{2}$ is given by

$$
\rho^{\prime}=\operatorname{Tr}_{Q_{1} C}\left[U\left(|\psi\rangle_{11}\langle\psi|\otimes| 0\rangle\langle 0|\otimes| g\rangle_{22}\langle g|\right) U^{\dagger}\right],
$$

with $U$ unitary time evolution operator for $Q_{1} C_{2}$, determined by the above described quantum protocol. We consider sudden switch on/off of the couplings, i.e. $f_{1}(t)=1$ for $0 \leq t \leq \tau, f_{1}(t)=0$ otherwise; $f_{2}(t)=1$ for $\tau \leq t \leq 2 \tau, f_{2}(t)=0$ otherwise. The quality of state transfer is measured by the fidelity

$$
F=\left\langle\psi\left|\rho^{\prime}\right| \psi\right\rangle
$$

In the Jaynes-Cummings limit, the state transfer is perfect and the fidelity $F=1$.

\section{Results}

The fidelity $F$ as a function of the coupling strength $g$ is shown in Figure 1 (left plot, full curve), for a specific state $|\psi\rangle$, while the dependence of $F$ on the initial state can be seen (for a value of $g$ in the 
USC regime) in the right plot of the same figure. The state transfer is perfect $(F=1)$ in the RWA limit $g \rightarrow 0$. In the ultrastrong coupling regime $(g>0.1), F$ drops significantly. Moreover, the fidelity is a non-monotonic function of the coupling strength, with maxima at $g_{k}^{(M)} \approx \omega /(2 k+1)$ and minima at $g_{k}^{(m)} \approx \omega /(2 k)(k=1,2, \ldots ; \omega=1$ in our units). This regular structure is a consequence of the terms beyond the RWA in Hamiltonian (1). Indeed, the Bloch vector (of $Q_{1}$ when $Q_{1}$ and $C$ are coupled or of $Q_{2}$ when the interaction is between $Q_{2}$ and $C$ ) rotates with a speed oscillating with frequency $2 \omega$ and therefore also the distance between the exact and the RWA evolution exhibits oscillations of frequency $2 \omega[26]$.

The strong connection between the fidelity decay and the DCE is witnessed by the fact that the fidelity exhibits anticorrelation of peaks and valleys with the mean number $\langle n\rangle$ of photons generated in the cavity, both at the end of the protocol (dashed curve in the left plot of Figure 1) and for the "pure" DCE (dot-dashed curve in the same figure). In the latter case, qubit $Q_{2}$ and the cavity $C$ are prepared in their ground state and the evolution of system $Q_{1} C$ is followed up to time $\tau$. Note that the evolution of a generic input state for $\mathrm{Q}_{1}$ also includes the evolution of the non-interacting ground-state $|g\rangle_{1}|0\rangle$, that is, the pure DCE. The photons generated by the pure DCE can lead to further, stimulated emission of photons but also to the coherent destruction of photons (anti-DCE [27-29]).
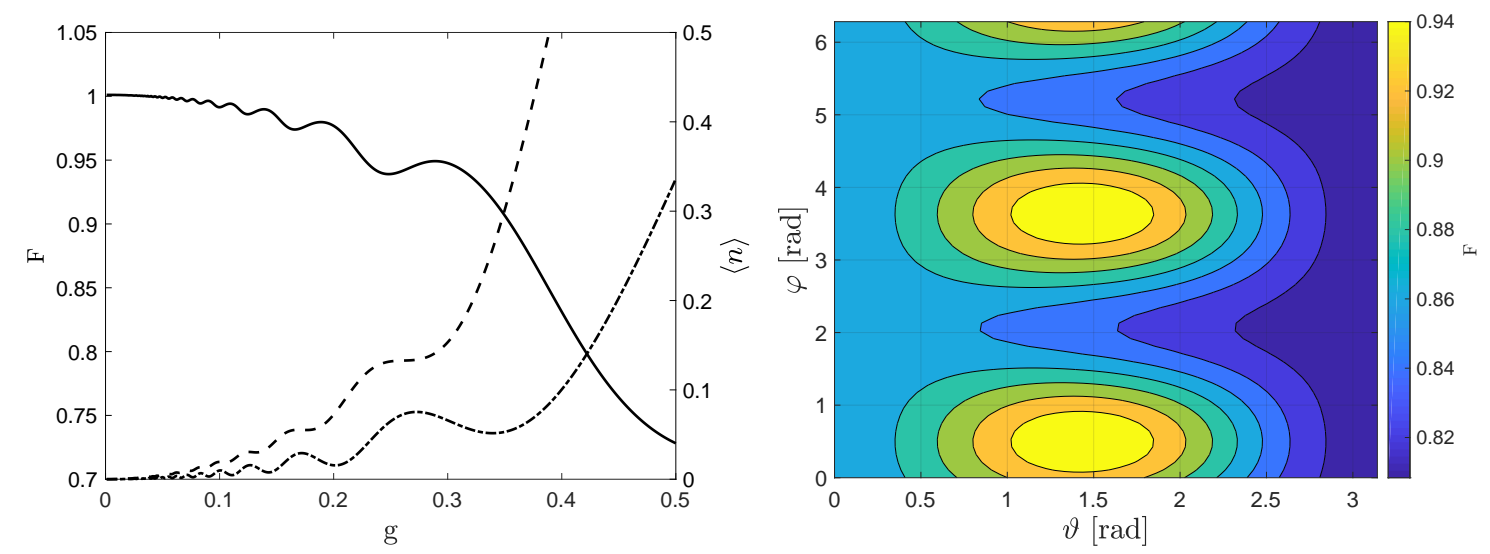

Figure 1. (Left) for the initial state $|\psi\rangle=\sqrt{0.2}|g\rangle+i \sqrt{0.8}|e\rangle$, fidelity $F$ (full curve, left axis) and mean photon number $\langle n\rangle$ (right axis, dashed curve) as a function of the qubit-cavity coupling strength $g$. The mean photon number is also shown for the pure DCE (dot-dashed curve). (Right) Contour plot for fidelity $F$ as a function of the Bloch sphere angles $\theta$ and $\varphi$ for the input state, at $g=0.4$.

In the right plot of Figure 1, we show the dependence of the fidelity $F$ for the Rabi transfer protocol on the initial state, $|\psi\rangle=\cos (\theta / 2)|g\rangle+e^{i \varphi} \sin (\theta / 2)|e\rangle$, for $g=0.4$. While in this regime the fidelity is in general significantly deteriorated $(F \approx 0.8-0.9)$, a non trivial dependence on the Bloch sphere angles $\theta$ and $\varphi$ can be seen.

\section{Discussion}

We have shown that the DCE severely limits the performance of quantum information protocols in the ultra-strong coupling regime, even in the ideal case considered here where dynamics is not affected by dissipation. While the results presented above are for the state transfer protocol, we have shown in [3] that more generally the DCE puts an intrinsic limit to the capability of the Rabi-based protocols to transmit quantum information. Novel schemes are required in order to counteract the DCE. Preliminary results [30] with a protocol inspired by stimulated Raman adiabatic passage (STIRAP) [31] show enhanced reliability of state transfer up to relatively large $g \approx 0.2$, also in presence of cavity damping. Optimal control techniques [16,32] could also be applied to this problem, with foreseen further improvements for the reliability of quantum protocols in the USC regime.

Acknowledgments: We acknowledge support by the INFN through the project "QUANTUM". 


\section{References}

1. Forn-Díaz, P.; García-Ripoll, J.J.; Peropadre, B.; Orgiazzi, J.-L.; Yurtalan, M.A.; Belyansky, R.; Wilson, C.M.; Lupascu, A. Ultrastrong coupling of a single artificial atom to an electromagnetic continuum in the nonperturbative regime. Nat. Phys. 2017, 13, 39.

2. Yoshihara, F.; Fuse, T.; Ashhab, S.; Kakuyanagi, K.; Saito, S.; Semba, K. Superconducting qubit-oscillator circuit beyond the ultrastrong-coupling regime. Nat. Phys. 2017, 13, 44.

3. Benenti, G.; D'Arrigo, A.; Siccardi, S.; Strini, G. Dynamical Casimir effect in quantum-information processing. Phys. Rev. A 2014, 90, 052313.

4. Dodonov, V.V. Current status of the dynamical Casimir effect. Phys. Scr. 2010, 82, 038105.

5. Nation, P.D.; Johansson, J.R.; Blencowe, M.P.; Nori, F. Colloquium: Stimulating uncertainty: Amplifying the quantum vacuum with superconducting circuits. Rev. Mod. Phys. 2012, 84, 1.

6. de Liberato, S.; Gerace, D.; Carusotto, I.; Ciuti, C. Extracavity quantum vacuum radiation from a single qubit. Phys. Rev. A 2009, 80, 053810.

7. Jaskula, J.-C.; Partridge, G.B.; Bonneau, M.; Lopes, R.; Ruaudel, J.; Boiron, D.; Westbrook, C.I. Acoustic analog to the dynamical Casimir effect in a Bose-Einstein condensate. Phys. Rev. Lett. 2012, 109, 220401.

8. Benenti, G.; Siccardi, S.; Strini, G. Exotic states in the dynamical Casimir effect. Eur. Phys. J. D 2014, 68, 139.

9. Koghee, S.; Wouters, M. Dynamical Casimir emission from polariton condensates. Phys. Rev. Lett. 2014, 112, 036406.

10. Felicetti, S.; Sanz, M.; Lamata, L.; Romero, G.; Johansson, G.; Delsing, P.; Solano, E. Dynamical Casimir Effect Entangles Artificial Atoms. Phys. Rev. Lett. 2014, 113, 093602.

11. Benenti, G.; Strini, G. Dynamical Casimir effect and minimal temperature in quantum thermodynamics. Phys. Rev. A 2015, 91, 020502(R).

12. Sabín, C.; Fuentes, I.; Johansson, G. Quantum discord in the dynamical Casimir effect. Phys. Rev. A 2015, 92, 012314.

13. Sabín, C.; Adesso, G. Generation of quantum steering and interferometric power in the dynamical Casimir effect. Phys. Rev. A 2015, 92, 042107.

14. Stassi, R.; de Liberato, S.; Garziano, L.; Spagnolo, B.; Savasta, S. Quantum control and long-range quantum correlations in dynamical Casimir arrays. Phys. Rev. A 2015, 92, 013830.

15. Angaroni, F.; Benenti, G.; Strini, G. Reconstruction of electromagnetic field states by a probe qubit. Eur. Phys. J. D 2016, 70, 225.

16. Hoeb, F.; Angaroni, F.; Zoller, J.; Calarco, T.; Strini, G.; Montangero, S.; Benenti, G. Amplification of the parametric dynamical Casimir effect via optimal control. Phys. Rev. A 2017, 96, 033851.

17. Macrì, V.; Ridolfo, A.; di Stefano, O.; Kockum, A.F.; Nori, F.; Savasta, S. Nonperturbative dynamical Casimir effect in optomechanical systems: Vacuum Casimir-Rabi splittings. Phys. Rev. X 2018, 8, 011031.

18. Angaroni, F.; Benenti, G.; Strini, G. Applications of Picard and Magnus expansions to the Rabi model. Eur. Phys. J. D 2018, 72, 188.

19. Blais, A.; Huang, R.-S.; Wallraff, A.; Girvin, S.M.; Schoelkopf, R.J. Cavity quantum electrodynamics for superconducting electrical circuits: An architecture for quantum computation. Phys. Rev. A 2004, 69, 062320.

20. Wallraff, A.; Schuster, D.I.; Blais, A.; Frunzio, L.; Huang, R.-S.; Majer, J.; Kumar, S.; Girvin, S.M.; Schoelkopf, R.J. Strong coupling of a single photon to a superconducting qubit using circuit quantum electrodynamics. Nature 2004, 431, 162.

21. Sillanpää, M.A.; Park, J.I.; Simmonds, R.W. Coherent quantum state storage and transfer between two phase qubits via a resonant cavity. Nature 2007, 449, 438.

22. Plastina, F.; Falci, G. Communicating josephson qubits. Phys. Rev. B 2003, 67, 224514.

23. Meystre, P.; Sargent, M., III. Elements of Quantum Optics, 4th ed.; Springer: Berlin, Germany, 2007.

24. Benenti, G.; Casati, G.; Rossini, D.; Strini, G. Principles of Quantum Computation and Information, 2nd ed.; World Scientific: Singapore, 2019, in press.

25. Braak, D.; Chen, Q.-H.; Batchelor, M.T.; Solano, E. Semi-classical and quantum Rabi models: In celebration of 80 years. J. Phys. A Math. Theor. 2016, 49, 300301.

26. Benenti, G.; Siccardi, S.; Strini, G. Nonperturbative interpretation of the Bloch vector's path beyond the rotating-wave approximation. Phys. Rev. A 2013, 88, 033814. 
27. de Sousa, I.M.; Dodonov, A.V. Microscopic toy model for the cavity dynamical Casimir effect. J. Phys. A Math. Theor. 2015, 48, 245302.

28. Veloso, D.S.; Dodonov, A.V. Prospects for observing dynamical and anti-dynamical Casimir effects in circuit QED due to fast modulation of qubit parameters. J. Phys. B At. Mol. Opt. Phys. 2015, 48, 165503.

29. Motazedifard, A.; Naderi, M.H.; Roknizadeh, R. Analogue model for controllable Casimir radiation in a nonlinear cavity with amplitude-modulated pumping: Generation and quantum statistical properties. J. Opt. Soc. Am. B 2015, 32, 1555.

30. Stramacchia, M.; Ridolfo, A.; Benenti, G.; Paladino, E.; Pellegrino, F.M.D.; Falci, G. Speedup of Adiabatic Multiqubit State-Transfer by Ultrastrong Coupling of Matter and Radiation. Proceedings 2019, 12, 35, doi:10.3390/proceedings2019012035.

31. Vitanov, N.V.; Rangelov, A.A.; Shore, B.W.; Bergmann, K. Stimulated Raman adiabatic passage in physics, chemistry, and beyond. Rev. Mod. Phys. 2017, 89, 015006.

32. Rach, N.; Müller, M.M.; Calarco, T.; Montangero, S. Dressing the chopped-random-basis optimization: A bandwidth-limited access to the trap-free landscape. Phys. Rev. A 2015, 92, 062343.

(C) 2019 by the authors. Licensee MDPI, Basel, Switzerland. This article is an open access article distributed under the terms and conditions of the Creative Commons Attribution (CC BY) license (http:// creativecommons.org/licenses/by/4.0/). 